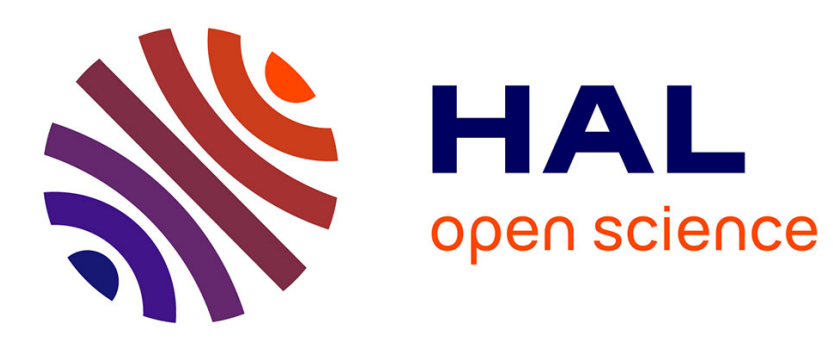

\title{
Etude d'une série chronologique de rendements en canne à sucre obtenus en Guadeloupe à partir d'un modèle de simulation du bilan hydrique
}

\author{
Sylvain Pellerin
}

\section{- To cite this version:}

Sylvain Pellerin. Etude d'une série chronologique de rendements en canne à sucre obtenus en Guadeloupe à partir d'un modèle de simulation du bilan hydrique. Agronomie, 1986, 6 (1), pp.91-98. hal-00884852

\section{HAL Id: hal-00884852 \\ https://hal.science/hal-00884852}

Submitted on 1 Jan 1986

HAL is a multi-disciplinary open access archive for the deposit and dissemination of scientific research documents, whether they are published or not. The documents may come from teaching and research institutions in France or abroad, or from public or private research centers.
L'archive ouverte pluridisciplinaire HAL, est destinée au dépôt et à la diffusion de documents scientifiques de niveau recherche, publiés ou non, émanant des établissements d'enseignement et de recherche français ou étrangers, des laboratoires publics ou privés. 


\title{
Etude d'une série chronologique de rendements en canne à sucre obtenus en Guadeloupe à partir d'un modèle de simulation du bilan hydrique
}

\author{
Sylvain PELLERIN \\ I.N.R.A., Station d'Agronomie, Centre de Recherches des Antilles et de la Guyane
} F.W.I. - 97170 Petit-Bourg (Guadeloupe)

RÉSUMÉ

L'examen des rendements bruts (matière fraîche) obtenus depuis 1952 dans le plus important domaine cannier de la Grande-Terre (Guadeloupe) fait apparaître une forte variabilité interannuelle et une tendance à la baisse. Les caractéristiques du milieu physique (climat tropical à saison sèche marquée et sols argileux) et l'absence $a$ priori de facteurs limitants techniques suggèrent le caractère limitant de l'alimentation en eau.

Pour tester dans quelle mesure la variabilité des rendements bruts est la conséquence des irrégularités pluviométriques, un modèle de simulation du bilan hydrique est proposé. Les rendements sont confrontés au rapport ETR/ETM calculé sur le cycle de la canne.

Entre 1964 et 1984, on rend compte des rendements bruts obtenus par la seule variabilité climatique.

La chute des rendements se situe entre 1963 et 1964, date qui coïncide avec l'introduction des cane-loaders et l'adoption du brûlage avant récolte. Plusieurs interprétations possibles sont proposées.

Mots clés additionnels : Vertisol.

Analysis of a chronological series of sugar cane yields (Guadeloupe, F.W.I.) using a simulated water balance model.

In the main sugar cane area of "Grande Terre" (Guadeloupe ; French West Indies) a survey of fresh matter yields obtained since 1952 showed great variability and a tendency for decrease. The climate of the area is tropical with a pronounced dry season. Soils are characterized by a high content of swelling clay minerals (vertisols). Technical practices seemed to be adequate and water nutrition was probably the main limiting factor for cane growth. In order to test whether fresh matter yield variability could be explained by rainfall irregularities a simulated model of water balance is proposed, comparing yields to ETR/ETM ratio calculated during the sugar cane growth period. From 1964 fresh matter yields could be explained by climatic variability only. There was, however, a clear yield decrease in 1963/1964, corresponding to the moment when cane loaders were introduced and burning before cropping was first practised. The effects of this on sugar cane yields are discussed.

Additional key words : Vertisol.

TABLEAU 1

Liste des symboles.

List of symbols.

$c$

I ГР (mm)

$\operatorname{ETM}(\mathrm{mm})$

$\operatorname{ETR}(\mathrm{mm})$

$\mathrm{EE}(\mathrm{mm})$

$\mathrm{P}(\mathrm{mm})$

R (mm)

$\mathrm{Rg}$ (Méga.loule/ $\mathrm{m}^{2}$ )
$=$ Indice des valeurs cumulées

$=$ Evapotranspiration potentielle

$=$ Evapotranspiration maximale

$=$ Evapotranspiration réelle

$=$ Excès d'eau

$=$ Indice journalier

$=$ Pluie

= Niveau de remplissage de la réserve utile

= Rayonnement global
Rgo (MégaJoule $/ \mathrm{m}^{2}$ ) = Rayonnement potentiel

RU (mm) = Réserve d'eau utile

$\mathrm{RFU}(\mathrm{mm}) \quad$ Réserve d'eau facilement utilisable

SS (heures) $\quad=$ Durée d'insolation

SSo (heures) $\quad=$ Durée astronomique du jour

w $(\%)$

Wc $(\%)$

$\mathrm{W}_{\mathrm{pF} 4.2}(\%)$

$=$ Humidité pondérale du sol

= Humidité à la capacité au champ

$=$ Humidité au point de flétrissement permanent 


\section{INTRODUCTION}

Les surfaces en canne à sucre, production traditionnelle de la Guadeloupe, sont actuellement en régression, entraînant par là une grave crise de l'industrie sucrière locale.

A cette diminution des surfaces s'ajoute, selon certains, une baisse des tonnages produits par hectare. De fait, l'évolution des rendements obtenus dans le plus important domaine cannier de la Grande-Terre $\left(^{1}\right)$, principale région productrice, révèle :

- une forte variabilité interannuelle,

- une apparente tendance à la baisse (fig. 1).

Les modifications importantes apportées depuis 30 ans à la conduite de la culture ont surtout concerné la mécanisation de la chaîne de récolte :

- introduction des cane-loaders en 1964 (coupe manuelle et ramassage mécanique) et adoption du brûlage avant récolte qui, en éliminant les feuilles sèches, facilite leur utilisation. A la même date, il y a eu modification de la conduite des cannes plantées, concernant environ 1/6 des surfaces chaque année, les cannes étant gardées en moyenne 6 ans : récolte au bout de 12 mois (plantation entre mars et juillet, récolte l'année suivante) au lieu de 18 mois (plantation entre juillet et septembre, récolte 18 mois plus tard) ;

- introduction des récolteuses mécaniques en 1974 et remodelage des terrains pour faciliter leur utilisation. Le brûlage a été maintenu jusqu'en 1980 .

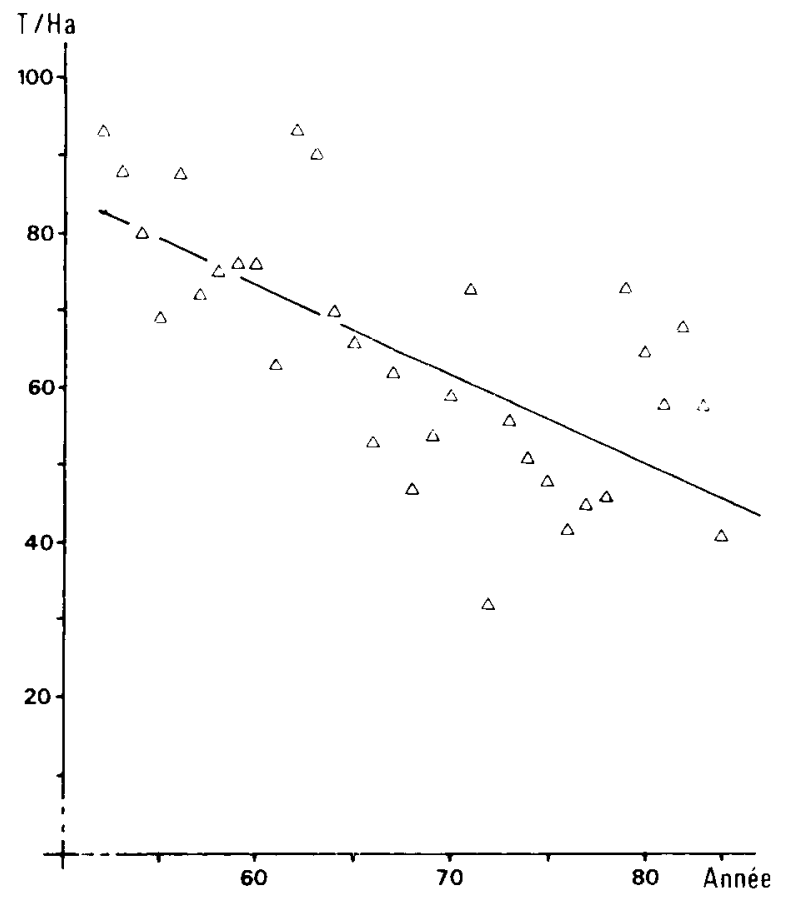

Figure 1

Evolution des rendements en canne à sucre obtenus dans le domaine de Gardel depuis 1952.

$R d t(t / h a)=-1,13$ (année -1952$)+82,2 \quad\left(r^{2}=0,46\right)$.

Sugar cane yields obtained on the Gardel estate since 1952.

(1) Ces domaines, exploités par les usines sucrières elles-mêmes, sont les Seuls pour lesquels on dispose d'informations sur les rendements. Ils représentaient en 1983,30 p. 100 de la production.
Plusieurs hypothèses, attribuant la baisse constatée des rendements à ces innovations techniques ont été avancées : tassement des sols par les engins, baisse de fertilité due aux opérations de remodelage ou à l'adoption du brûlage, ce qui réduit les restitutions organiques, ou encore suppression, du fait du brûlage, de l'effet mulch qu'assuraient les résidus de récolte.

Aucune explication vérifiée n'a été fournie. $\mathrm{Au}$ préalable, une analyse approfondie de la série chronologique des rendements obtenus au domaine Gardel était nécessaire, ce qui est l'objet de cet article.

Une étude des caractéristiques du milieu et des techniques culturales nous a orienté vers la construction d'un modèle de simulation du bilan hydrique pour examiner quelle est la part de l'irrégularité des rendements liée à la variabilité climatique interannuelle.

\section{MATÉRIEL ET MÉTHODES}

\section{A. Milieu physique et techniques culturales mises en ceuvre}

\section{Le climat}

Le climat est de type tropical, à saison sèche marquée. La pluviométrie moyenne annuelle est de $1300 \mathrm{~mm}$, avec une forte variabilité interannuelle $(\sigma=390 \mathrm{~mm})$. Le déficit hydrique $(\mathrm{ETP}>\mathrm{P})$ dure de janvier à août et atteint $500 \mathrm{~mm}$ en année normale (fig. 2).

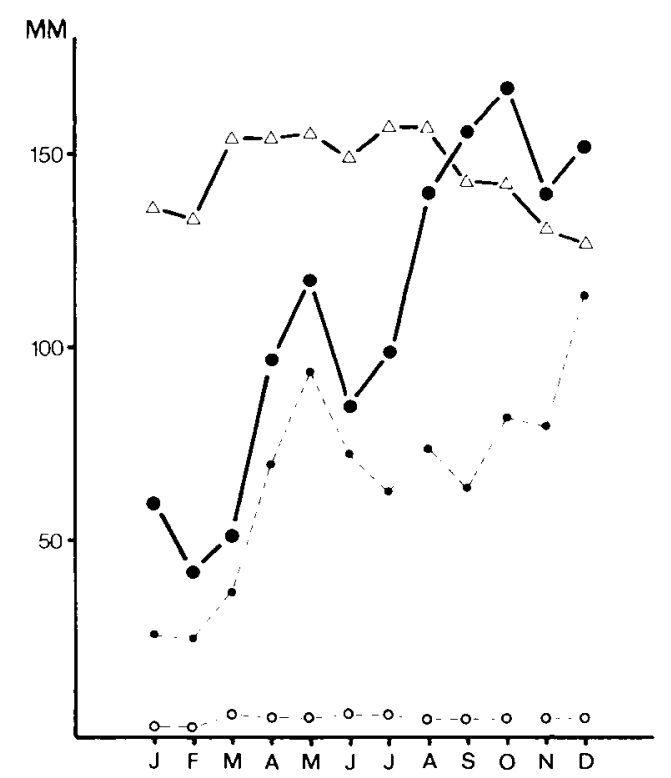

Figure 2

Pluviométries et évapotranspirations moyennes mensuelles.

Monthly rainfall and potential evapotranspiration.

- - Pluviométries moyennes mensuelles (Gardel, 1967-1983); Monthly rainfall (Gardel, 1967-1983).

-.-.-Ecarts-types; Standard deviation.

$\triangle-\triangle$ Evapotranspirations movennes mensuelles (Saint-François 1967-1983, $7 \mathrm{~km}$ de Gardel) ; Monthly potential evapotranspiration (Saint-François 1967-1983, $7 \mathrm{~km}$ from Gardel).

○----- $\mathrm{O}$ Ecarts-types; Standard deviation. 


\section{Les terrains}

Les sols sont argileux, développés sur calcaire madréporique et organisés en séquences depuis les sols calciques mélanisés peu profonds des hauts de pente jusqu'aux vertisols hydromorphes profonds de bas de pente.

Leur statut chimique est favorable : forte capacité d'échange cationique, teneurs élevées en bases échangeables avec dominance de $\mathrm{Ca}$ et $\mathrm{Mg}$ (COLMET DAAGE, 1969). Aucun apport d'amendement n'est nécessaire.

Leur richesse en argiles gonflantes leur confère par contre certaines propriétés physiques défavorables :

- plasticité et adhésivité élevées à l'état humide, forte cohésion à l'état sec,

- faible conductivité hydraulique après fermeture par gonflement de la macroporosité fissurale et tendance à l'hydromorphie.

\section{Techniques culturales mises en cuvre}

Les techniques culturales actuellement mises en œuvre dans les terres de l'usine Gardel sont calquées sur les recommandations du Centre Technique de la Canne et du Sucre (CTCS) et apparaissent adaptées à ce pédoclimat. Elles n'introduisent pas a priori de facteur limitant :

- en général le travail du sol est effectué sur sol $\mathrm{sec}$, en période de déficit (mai à juin) donc, a priori, sans risque de dégradation de l'état structural. La fragmentation est assurée par un sous-solage et 2 labours croisés séparés par un temps de fissuration sous l'action du climat;

- la lutte contre les adventices intervient depuis la plantation jusqu'à recouvrement total par la culture (herbicides de pré- et post-émergence, sarclage mécanique en interrang et arrachage manuel des graminées à souche résistante) ;

- la fertilisation apportée est censée couvrir les besoins d'une canne produisant 75 t/ha (d'après CTCS, 1982).

Les principales modifications techniques intervenues depuis 30 ans ont été rappelées en introduction. Il n'y a eu ni tendance à la diminution des doses d'engrais, ni moindre lutte contre les adventices. Des variétés améliorées ont été introduites progressivement chaque année dans les replantations. Les critères de choix ont surtout été la richesse en sucre et l'adaptation à la récolte mécanique (NOIRTIN, comm. pers., 1984).

\section{Conclusion}

En l'absence de facteur limitant technique, l'alimentation hydrique apparaît comme le principal facteur limitant des rendements en canne. On observe de fait une corrélation positive assez lâche entre les rendements obtenus et la pluviométrie cumulée sur le cycle (au moins jusqu'à $1500 \mathrm{~mm}$ ) (fig. 3). Dès lors, pour tester dans quelle mesure l'irrégularité des rendements est liée à la variabilité climatique interannuelle, la construction d'un modèle de simulation du bilan hydrique était nécessaire.

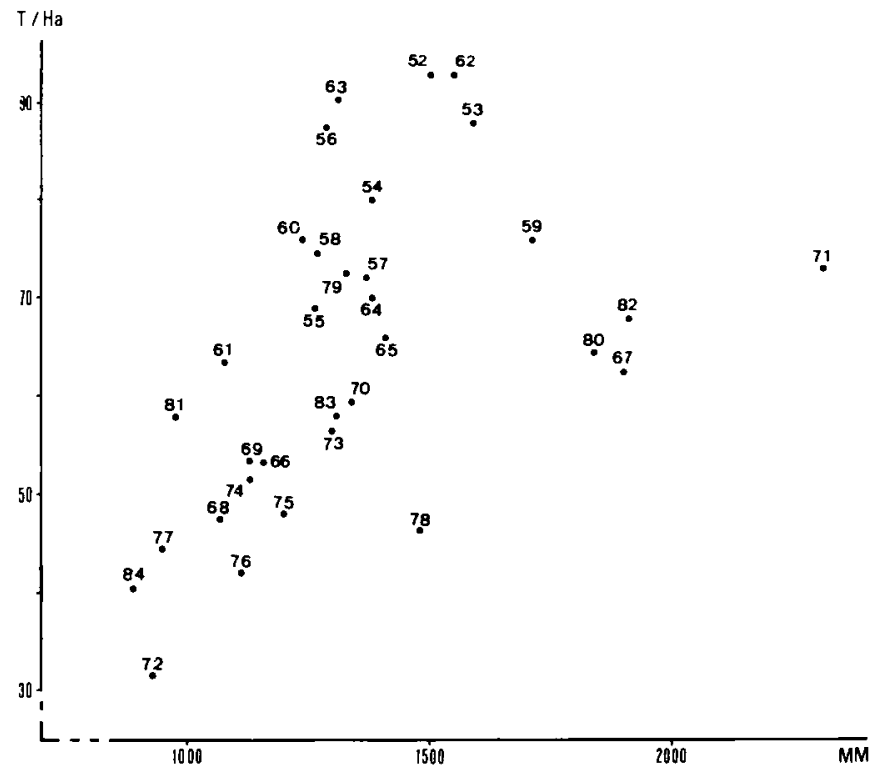

Figure 3

Rendements en canne obtenus sur le domaine de Gardel en fonction de la pluviométrie cumulée sur la période du ler avril au 31 mars.

Yield of sugar cane obtained on the Gardel estate in relation to cumulative rainfall for $I^{\text {st }}$ April-3I March.

\section{B. Le modèle}

\section{Principe et bases théoriques}

Plusieurs auteurs ont montré l'existence d'une relation approximativement linéaire entre la matière sèche fabriquée par un couvert végétal en situation d'alimentation hydrique limitante et le rapport ETR/ETM calculé sur le cycle (ROBELIN, 1962 ; BOUCHET, 1965 ; PUECH \& HERNANDEZ, 1973 ; MORIZET el al., 1984).

$\mathrm{Si}$, dans le cas étudié, l'eau a été facteur limitant, on doit s'attendre à retrouver cette relation.

Le modèle construit pour tester l'existence de cette liaison est schématisé en Annexe I.

Les données suivantes sont entrées jour par jour : pluviométrie $\left(P_{j}\right)$, rayonnement global $\left(R_{j}\right)$ et niveau de remplissage de la réserve utile du sol $\left(R_{j}\right)$.

Le modèle en déduit les valeurs de :

- l'ETP, calculée à partir du rayonnement global d'après un ajustement statistique établi localement,

- l'ETM, fonction de l'ETP et de l'importance des surfaces évaporantes. Le rapport ETM/ETP est estimé en fonction de l'âge de la canne, d'après des coefficients établis aux Hawaii (CHANG et al., 1965 ; JONES, 1980) dont les caractéristiques climatiques sont proches de celles de la Guadeloupe. Les résultats utilisés sont confirmés par d'autres travaux effectués en Afrique du Sud et à l'île Maurice (THOMPSON, 1965 ; HARDY, 1966, 1969),

- l'ETR, fonction de l'ETM et de l'état hydrique du sol. Tant que la RFU n'est pas épuisée on considère que l'intervention de la régulation stomatique est négligeable $\left(\frac{E T R}{E T M}=1\right)$. Pour les humidités inférieures, le rapport ETR/ETM diminue linéairement (HALLAIRE, 1960 ; AHO et al., 1980) jusqu'à s'annuler au point de flétrissement permanent $\left(\mathrm{W}=\mathrm{W}_{\mathrm{pF} 4.2}\right)$. 
L'effet dépressif des excès d'eau $\left(w>W_{c}\right)$ sur le rapport ETR/ETM (AHO et al., 1980) n'a pas été pris en compte dans le modèle, faute de références.

Ces valeurs permettent de calculer le niveau de remplissage de la réserve utile pour le jour suivant : $\mathbf{R}_{\mathrm{j}+1}=\mathbf{R}_{\mathbf{j}}+\mathbf{P}_{\mathrm{j}}-\mathrm{ETR}_{\mathrm{j}}$. Si, par ce calcul, $\mathbf{R}_{\mathbf{j}+1}$ est supérieur à la capacité de la réserve utile, soit $R U$, on pose $R_{j+1}=R U$. L'excédent est comptabilisé comme excès d'eau (EE) et est supposé évacué, par drainage ou ruissellement. En l'absence d'excès d'eau par contre, les pertes par drainage ou ruissellement sont supposées négligeables.

Sur le cycle de la canne on en déduit :

- le rapport ETR/ETM cumulé,

- l'importance et la date des déficits hydriques (ETM - ETR) et des excès d'eau (EE),

- le niveau de remplissage de la réserve utile en fin de cycle, reporté pour le calcul sur le cycle suivant.

\section{Estimation des valeurs de $R U$, de $R F U$ et humi- dités caractéristiques $\left(\mathrm{W}_{\mathrm{pF} 4.2} ; \mathrm{W}_{\mathrm{c}}\right)$}

La détermination de ces valeurs en vertisol, présente des difficultés spécifiques :

- l'humidité du sol, à un pF donné, dépend fortement de son histoire hydrique (TESSIER \& PEDRO, 1980 ; TESSIER et al., 1980),

- les techniques classiques de détermination des humidités caractéristiques sont perturbées par les propriétés du matériau,

- enfin, du fait des très faibles vitesses de transfert, la quantité d'eau disponible pour la culture est extrêmement dépendante de la densité racinaire.

Les seules références existantes pour une culture de canne ont été établies in situ par confrontation des courbes de croissance et d'évolution simulée de l'état hydrique du sol. La RFU serait de l'ordre de $85 \mathrm{~mm}$ en vertisol profond comme en sol peu profond sur calcaire poreux (KOKASON, 1982 ; VALANCOGNE et al., 1984). Ces 2 types de sol sont de loin les plus représentés sur le domaine Gardel. Nous avons donc retenu cette valeur.

Un calcul approximatif a permis d'estimer à environ $150 \mathrm{~mm}$ la RU moyenne des sols présents sur le domaine. Cette valeur a été calculée pour une profondeur d'enracinement de $1 \mathrm{~m}$, ce qui correspond d'après observation à l'épaisseur de sol fortement explorée par les racines. Dans le cas des sols peu profonds sur calcaire poreux, celui-ci est partiellement exploré par les racines et il contribue de ce fait à la réserve utile (VALANCOGNE et al., 1984).

\section{Utilisation du modèle}

La récolte de la canne s'étalant de février à mai ou juin, le rapport ETR/ETM a été calculé sur un cycle moyen allant du $1^{\text {er }}$ avril au 31 mars.

Les résultats sont confrontés aux rendements en matière fraîche calculés à partir du poids total récolté. On ne dispose ni de mesures des teneurs en matière sèche ni de données utilisables sur le tonnage de sucre récolté. L'emploi du critère rendement en matière fraîche, seul critère utilisable dans ces conditions, introduit un biais car il peut être affecté par les conditions pluviométriques qui précèdent la récolte.
Compte tenu de l'étalement des dates de récolte sur plusieurs mois et de l'importance des surfaces concernées (plus de $1000 \mathrm{ha}$ ), on peut cependant penser que la variabilité interannuelle des teneurs moyennes en eau des cannes récoltées doit être faible.

\section{RÉSULTATS ET DISCUSSION}

\section{A. Résultats}

Il n'apparaît pas de corrélation nette entre les rendements obtenus et les rapports ETR/ETM calculés sur le cycle.

Un examen attentif permet cependant de distinguer 2 périodes (fig. 4) :

1) De 1952 à 1962, les points sont alignés à une exception près (1957). Ce constat est convergent avec l'hypothèse que l'eau a été facteur limitant du rendement.

L'écart de l'année 1963 est dû à des reports sur pied effectués à la suite de la campagne 1962, pour laquelle il y a eu surproduction. En 1962, en effet, le quota de production de sucre autorisé pour la Guadeloupe a été atteint. Certaines parcelles d'usine non récoltées ont été gardées sur pied pour l'année suivante et ont poursuivi leur croissance, d'où les forts rendements 1963 .

2) De 1964 à 1984, les points sont, à quelques exceptions près, également alignés mais sur une droite

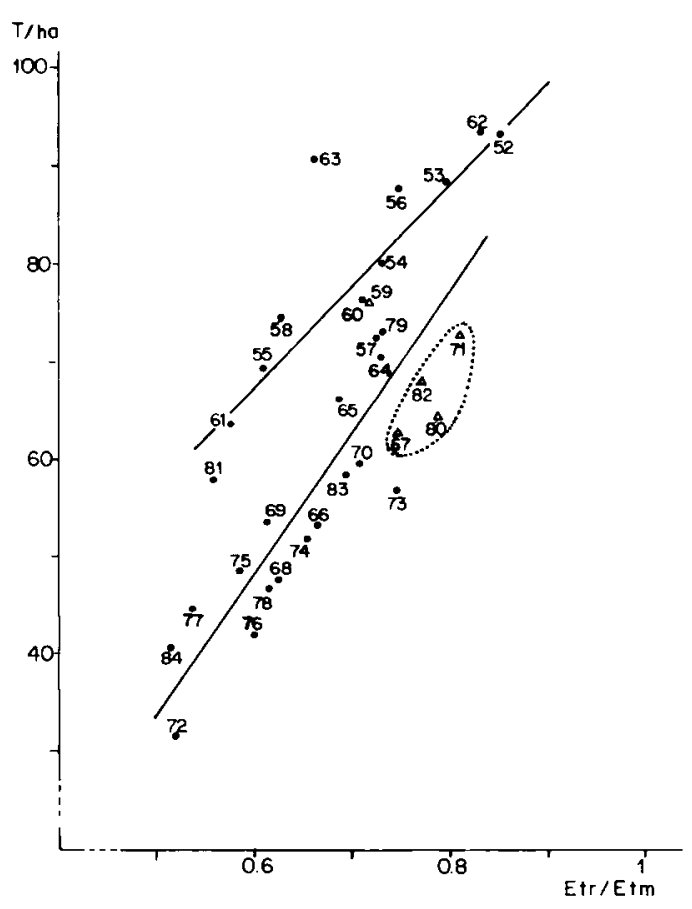

Figure 4

Rendements moyens en canne obtenus sur le domaine Gardel en fonction du rapport ETR/ETM calculé sur la période du ler avril au 31 mars.

$\triangle$ Années pour lesquelles l'excès d'eau calculé sur le cycle dépasse $500 \mathrm{~mm}$.

De 1952 à $1962:$ Rdt $(t / h a)=102,1$ ETR/ETM $+5,6 r^{2}=0,85$ De 1964 à 1984 (sauf 1967-71-73-80-81 et 82) : $R d t(t / h a)=143,6$ ETR/ETM $-38,5 r^{2}=0,85$.

Yield of sugar cane obtained on the Gardel estate and ETR/ETM ratio calculated for ${ }^{1 \text { st }}$ April-3l March.

$\triangle$ Years for which calculated water excess exceeded $500 \mathrm{~mm}$. 
en dessous de la précédente. Pour les points qui s'écartent on peut avancer les explications suivantes :

- l'année 1973 fait suite à une année exceptionnellement sèche. Cette sécheresse a entraîné la mort de certaines souches (NOIRTIN, comm. pers., 1984), d'où un handicap pour le rendement 1973 qui serait dû à un défaut de peuplement,

- les années 1967, 1971, 1980 et 1982, légèrement en dessous de la droite moyenne, ont été marquées par un fort excès d'eau (fig. 5). Dans ce type de sol, il peut en avoir résulté une moindre croissance de la canne, sensible à l'anoxie (GUMBS \& SIMPSON, 1981) et une nécrose du système racinaire, d'où une sensibilité accrue à d'éventuels déficits hydriques à venir,

- enfin l'année 1981 s'écarte également de la droite. Les raisons probables de cet écart seront discutées ci-après.

\section{B. Discussion}

1) Depuis 1964, l'irrégularité des rendements apparaît principalement liée à la variabilité climatique interannuelle :

Il n'y a pas eu chute des rendements lors de la mécanisation de la récolte et des opérations de remodelage (1974). Ce constat contredit une hypothèse parfois avancée pour expliquer la baisse des rendements. D'après les responsables de l'époque, les travaux de remodelage ont été faits prudemment au domaine Gardel, en évitant le décapage des sols peu profonds de haut de pente, ce qui tend à être confirmé par nos résultats.

Il n'y a pas eu non plus augmentation progressive des rendements malgré l'introduction chaque année de variétés améliorées. A cela deux explications possibles :

- la sélection variétale a surtout porté sur la richesse en sucre et l'aptitude à la récolte mécanique (port des tiges),

- dans cette zone de la Guadeloupe, les conditions hydriques limitantes empêchent l'extériorisation des potentialités génétiques.

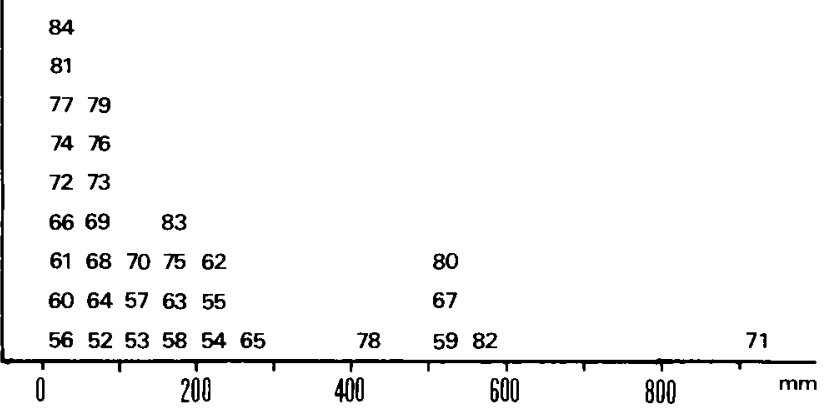

\section{Figure 5}

Distribution des années en fonction des excès d'eau cumulés sur le cycle de la canne.

Distribution of years for cumulative water excess over the sugar cane growth period.
2) La chute des rendements se situe entre 1963 et 1964, année qui coïncide avec l'introduction des caneloaders, l'adoption du brûlage avant récolte et la modification des durées de cycle sur canne plantée. Faute d'informations suffisantes ce constat est d'interprétation agronomique difficile.

- Il peut s'agir d'un artefact dû par exemple :

- à une augmentation des pertes entre coupe et pesée du fait de l'utilisation des cane-loaders (c'est peu probable puisqu'un glanage est pratiqué),

- à la suppression par le brûlage de résidus (feuilles, ...) antérieurement pesés avec la canne. On devrait alors observer une évolution des richesses en sucre mesurées, ce qui n'est pas le cas.

- La réduction des durées de cycle sur canne plantée a forcément entraîné une baisse des rendements. D'après des travaux effectués en Barbade sur plusieurs années et dans des conditions de milieu très proches de celles de la Grande-Terre, la différence de rendement $(\mathrm{Rdt})$, en $\mathrm{t} / \mathrm{ha}$, entre des cannes plantées de 12 mois et de 18 mois serait la suivante (RouSE, 1966) :

Rdt (canne 12 mois) $=0,7 \mathrm{Rdt}$ (canne 18 mois) +9 .

La baisse de rendement correspondante, affectant 1/6 des surfaces environ chaque année, ne suffit pas cependant pour rendre compte de la baisse constatée des rendements moyens.

- Enfin il se peut que la pratique du brûlage ait eu un effet néfaste sur la croissance des rejetons. En effet divers travaux ont mis en évidence un effet dépressif du brûlage en zone à saison sèche marquée, dû à la suppression de l'effet mulch qu'assurent les résidus de récolte (GOSNELL \& LONSDALE, 1977). Après dégradation, il se peut aussi que l'horizon de résidus organiques s'accumulant en surface d'année en année augmente de manière significative la capacité de rétention en eau. Les positions respectives des 2 droites, convergentes pour un rapport ETR/ETM proche de 1 et s'écartant d'autant plus que l'année est plus sèche, suggèrent de fait un problème de conservation de l'eau.

L'abandon du brûlage à partir de 1981 expliquerait la position de ce point, proche de la $1^{\text {re }}$ droite. En 1982, la croissance a été handicapée par de très forts excès d'eau en début de cycle ayant entraîné des chutes de rendements atteignant $30 \mathrm{t} / \mathrm{ha}$ sur vertisol profond (CABIDOCHE, 1985). Ceci expliquerait la position de ce point, très en dessous de la $1^{\text {re }}$ droite. En 1983 et 1984 enfin, du fait de la sécheresse, tous les résidus de récolte ont été ramassés par les petits éleveurs pour l'alimentation du bétail.

3) Pour chacune des 2 périodes, la variabilité résiduelle (écarts par rapport aux droites) peut être due à des raisons culturales (problèmes phytopathologiques par exemple) ou liée aux insuffisances du modèle, en particulier :

- d'éventuelles variations de sensibilité à la sécheresse au cours du cycle ne sont pas prises en compte,

- les durées réelles de cycle, liées aux variations interannuelles des dates de récolte ne sont pas connues. A cet égard signalons que, même s'il existe des fluctuations interannuelles, la durée de cycle en 
moyenne ne change pas et les écarts se compensent forcément d'une année sur l'autre. En regroupant les points par couples et en travaillant sur les moyennes on devrait donc atténuer cet effet. C'est ce qui a été fait sur la figure 6 , en éliminant les couples pour lesquels un des points présentait un caractère particulier déjà signalé. On obtient ainsi un meilleur alignement.

\section{CONCLUSION}

L'analyse d'une série chronologique de rendements telle que nous l'avons conduite procède d'une démarche agronomique et, en cela, se veut différente des approches statistiques parfois utilisées pour ce type d'investigation :

Une analyse agronomique préalable du milieu et des techniques utilisées en vue de détecter les facteurs limitants probables a souligné l'importance de l'alimentation hydrique. Dès lors, la construction d'un modèle de simulation du bilan hydrique devenait nécessaire pour tester dans quelle mesure l'irrégularité des rendements était liée à la variabilité climatique interannuelle. Ce modèle a été construit d'après un ensemble de connaissances sur les relations climat-solplante.

Les résultats ont confïrmé l'importance de l'alimentation hydrique comme variable explicative des rendements. La baisse constatée (fig. 1) a pu être située dans le temps. Certaines hypothèses explicatives ont pu être avancées, d'autres éliminées. On n'a pas observé d'augmentation des rendements liée à l'amélioration variétale. Cela est sans doute dû au fait que le critère pris en compte (rendement total) n'est pas celui sur lequel a le plus porté la sélection.

En l'absence de facteur limitant technique, et à condition de le vérifier pour d'autres sites, ce travail souligne l'intérêt des séries de rendements obtenus dans les domaines usiniers comme moyen de connaître les potentialités de rendement en canne. Compte tenu de la variabilité climatique interannuelle et des fortes hétérogénéités pédoclimatiques que recouvre son aire de culture, c'est une référence importante pour l'amélioration de la conduite de la canne.

Les limites de notre travail sont évidentes et désignent les améliorations à rechercher.

Faute de données sur le rendement en matière sèche ou en sucre, c'est le rendement en matière fraîche qui a été considéré. Il serait nécessaire de vérifier que la teneur en eau moyenne des cannes récoltées sur le domaine est peu variable entre années.

Le modèle utilisé, par construction, est imparfait, car forcément contingent du niveau des connaissances agronomiques sur lequel il se fonde. Plusieurs paramètres ont été estimés de façon approximative, les durées réelles de cycle et des caractéristiques propres à la plante ne sont pas prises en compte (d'éventuelles variations de sensibilité à la sécheresse au cours du cycle par exemple). Des contrôles au champ pour tester le modèle et des études plus spécifiques devraient permettre de l'améliorer.

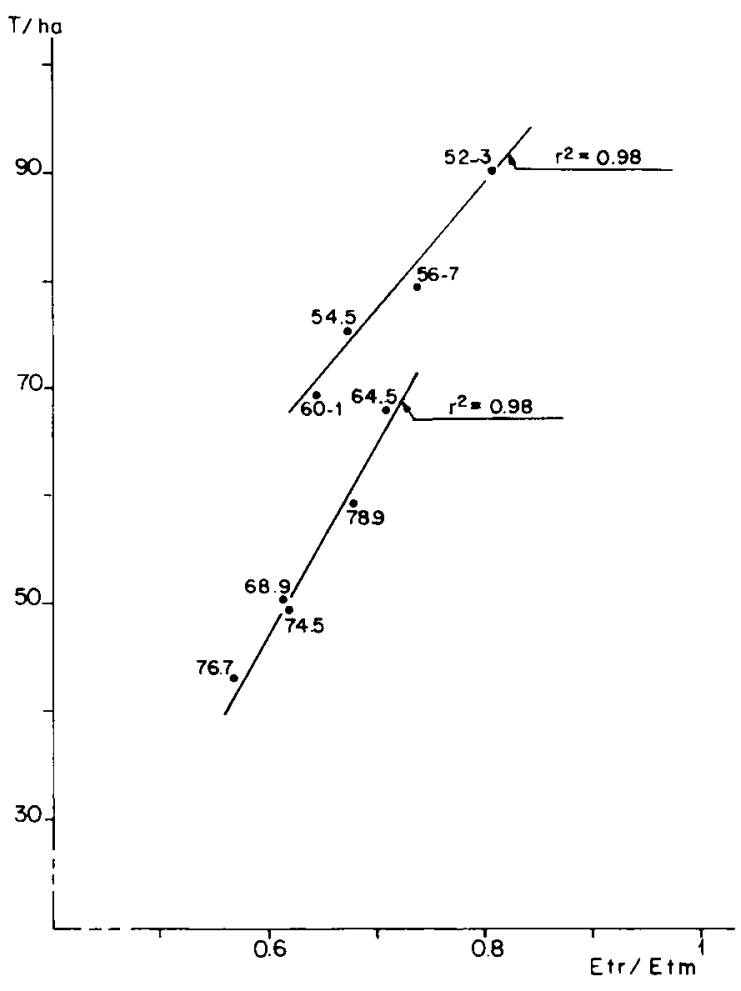

Figure 6

Moyenne des rendements, par couple de deux années consécutives, en fonction du rapport ETR/ETM moyen.

Mean yield, for pairs of consecutive years, related to mean ETR/ETM ratio.

\section{REMERCIEMENTS}

Nous remercions Messieurs HoPPFNER (ORSTOM Guadeloupe) et NorRTIN, ancien directeur de la sucrerie Gardel, pour les informations qu'ils nous ont communiquées. Merci aussi à R. BoNHomme (I.N.R.A., Bioclimatologie) el Y.-M. CABIDOCHE (I.N.R.A., Science $\mathrm{du}$ Sol) pour leurs avis et critiques.

ANNEXE I : Présentation du modèle

\section{1) Organigramme}

Celui-ci est présenté en figure 7

2) Origine des données climatiques (tabl. 2)

Certaines valeurs de rayonnement global manquantes ont été estimées à partir des durées d'insolation, d'après une formule établie localement :

$$
\operatorname{Rg}=\operatorname{Rg}_{0}\left(0,44 \frac{\mathrm{SS}}{\mathrm{SS}_{0}}+0,29\right)
$$

(Bonhomme \& VARLET-GranCHER, 1973).

3) Mode de calcul des valeurs d'évapotranspiration

\subsection{Calcul des ETP (ETP gazon)}

On a utilisé un ajustement statistique établi localement :

$$
\mathrm{ETP}=0,165 \mathrm{Rg}+1,5(\text { SCHOCH et al., 1975) } .
$$

Pour les années antérieures à 1967, pour lesquelles on ne dispose pas des mesures de rayonnement global, on a utilisé les valeurs moyennes journalières du rayonnement calculées sur la période 1967-1983. 


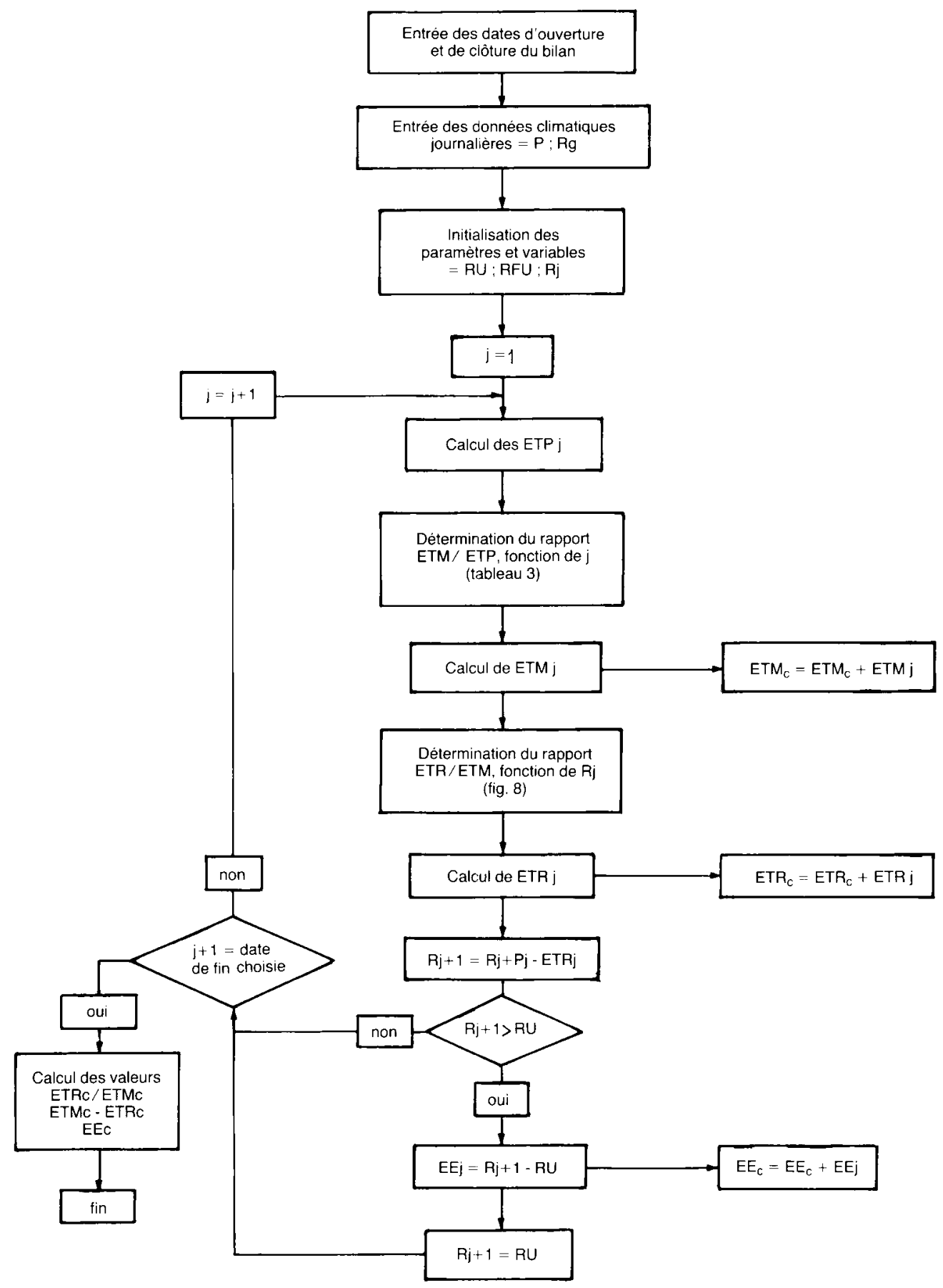

Figure 7

Organigramme du modèle.

Flow chart of the model.

TABLEAU 2

Origine des données climatiques.

Origin of climatic data.

\begin{tabular}{ccc}
\hline & Poste & Période d'enregistrement \\
\hline Pluviométrie & Gardel & $1938-1984$ \\
\hline $\begin{array}{c}\text { Rayonnement } \\
\text { global }\end{array}$ & $\begin{array}{c}\text { INRA-Ferme } \\
\text { de May }\end{array}$ & $1967-1984$ \\
\hline \hline
\end{tabular}

\subsection{Calcul des ETM}

On a utilisé les coefficients ETM/ETP établis par CHANG et al. (1965), en fonction de l'âge de la canne (tabl. 3). Ils ont été modifiés pour se rapporter à l'ETP gazon :

$\mathrm{ETM} / \mathrm{ETP}_{\text {gazon }}=\frac{1}{0,8} \mathrm{ETM} / \mathrm{ETP}$ bac classe A $(\mathrm{SCHOCH}$ et al., 1975) .

3.3. Calcul des ETR (fig. 8)

Pour $\mathrm{W}<\mathrm{w}<\mathrm{Wc}$ (RFU non vide) $: \mathrm{ETR} / \mathrm{ETM}=1$.

Pour $\mathrm{W}_{\mathrm{pF} 4.2}<\mathrm{w}<\mathrm{W}$ (RFU vide) :

$$
\mathrm{ETR} / \mathrm{ETM}=\frac{\mathrm{w}-\mathrm{W}_{\mathrm{pF} 4.2}}{\mathrm{~W}-\mathrm{W}_{\mathrm{pF} 4.2}}=\frac{\mathrm{R}}{\mathrm{RU}-\mathrm{RFU}} .
$$


TABLEAU 3

Valeur des coefficients ETM/ETP gazon, en fonction de l'âge de la canne.

$E T M / E T P$ ratios related to sugar cane age.

\begin{tabular}{lcccccc}
\hline Mois & 1 & 2 & 3 & 4 & 5 & 6 \\
\hline $\begin{array}{l}\text { ETM gazon } \\
\text { ETP }\end{array}$ & 0,5 & 0,55 & 0,6 & 0,8 & 1,1 & 1,2 \\
\hline Mois & 7 & 8 & 9 & 10 & 11 & 12 \\
\hline ETM gazon & 1,2 & 1,2 & 1,2 & 1,2 & 1,2 & 1,2 \\
\hline \hline
\end{tabular}

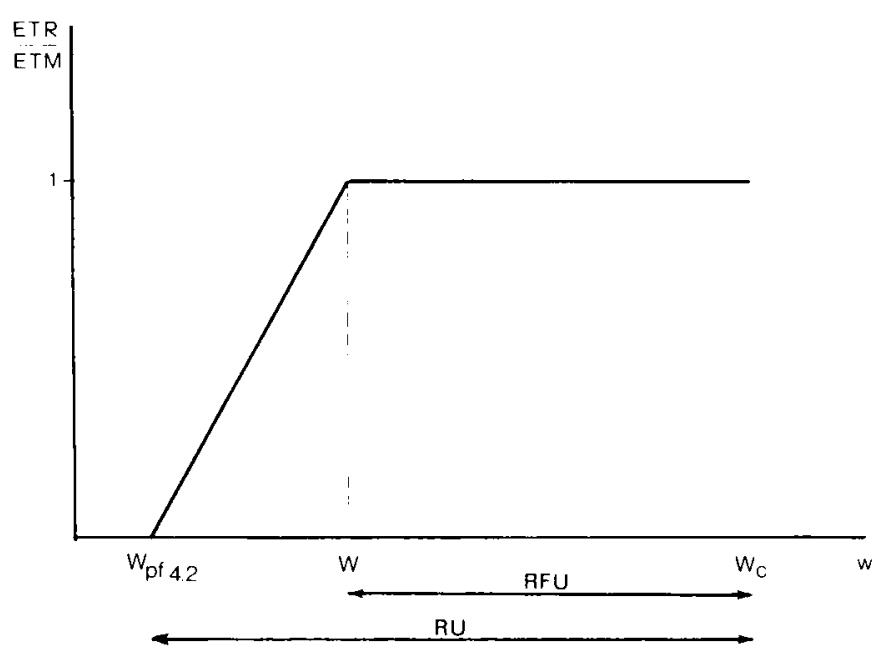

Figure 8

Evolution du rapport ETR/ETM, en fonction de l'état hydrique du sol.

ETR/ETM ratio related to water content of soil.

\section{RÉFÉRENCES BIBLIOGRAPHIQUES}

\begin{abstract}
Aho N., Daudet F. A., Vartanian N., 1980. Variations de la réserve facilement utilisable en eau du sol en relation avec différents facteurs écologiques. Ann. agron., 31 (2), 109-124.

Bonhomme R., Varlet-Grancher C., 1973. Evaluation de l'énergie solaire en Guadeloupe. In : "Le soleil au service de l'homme", Congrès UNESCO, Paris, E 125, 1-8.
\end{abstract}

Bouchet R. J., 1965. Rendement des cultures et déficit en eau. Bull. Tech. Inf. Min. Agric., 201, 539-551.

Cabidoche Y.-M., 1985. Distribution des sols à argiles gonflantes sur calcaire corallien (zone Caraibe). Utilisation de mesures de résistivité électrique. Comm. « Séminaire Franco-Cubain sur le fonctionnement des sols », La Havane, Cuba, 8-20 avril 1985. Ed. INRA-ORSTOM.

Chang J. H., Campbell R. B., Brodie H. W., Baver L. D., 1965. Evapotranspiration research at the HSPA experiment station. Proc. ISSCT, (12), 10-24.

Colmet Daage F., 1969. Aperçu sur les sols des Antilles. In : "Caribbean food crops society ". C.R. $7^{e}$ congrès annuel, Martinique-Guadeloupe, 4 juillet 1969, 242-250.

C.T.C.S., 1982. Le livret du planteur $-\mathrm{n}^{\circ} 1$ - La plantation et l'entretien des cannes, $20 \mathrm{p}$.

Gosnell J. M., Lonsdale J. E.., 1977. Effects of irrigation level and trash management on sugar cane. Int. sug. J., (80), 264-269 et 293303.

Gumbs F. A., Simpson L. A., 1981. Influence of flooding and soil moisture content on elongation of sugar cane in Trinidad. Expl. Agric. (17), 403-406.

Hallaire M., 1960. Le problème du potentiel de l'eau dans le sol et de la disponibilité de l'eau pour la végétation. Ann. Physiol. Vég., 2, $119-130$.

Hardy M., 1966. Water consumption of the cane plant (preliminary results of lysimeter experiments). MSIRI Annu. Rep., 91-102.

Hardy M., 1969. Observations on the evapotranspiration of sugar cane (final results of lysimeter experiments). MSIRI Annu. Rep., 104-106.
Jones C. A., 1980. A review of evapotranspiration studies in irrigated cane in Hawaii. Hawaian Planters Record, 59 (9), 195-213.

Kokason S., 1982. Croissance et état hydrique de la canne à sucre en relation avec les réserves en eau du sol. Mémoire de fin d'études. ISTOM, $93 \mathrm{p}$.

Morizet J., Robelin M., Baucher G., 1984. Résultats de 18 années d'observations lysimétriques sous climat limagnais. II. - Etude des relations entre l'eau et la production végétale. Agronomie, 4 (5), 407-416.

Puech J., Hernandez M., 1973. Evapotranspiration comparée de différentes cultures et étude de quelques facteurs influençant les rythmes de consommation. Ann. agron., 24 (4), 437-455.

Robelin M., 1962. Rapports entre augmentation de poids sec et transpiration en condition de sécheresse. C.R. Acad. Sci., Paris, 254, 718-720.

Rouse W. R., 1966. The moisture balance of Barbados and its influence on sugar cane yield. In : « Two Studies in Barbadian Climatology ». McGill University, Montreal, $54 \mathrm{p}$.

Schoch P. G., De Villele O., Granier J., 1975. Besoins climatiques en eau des cultures aux Antilles françaises. Nouv. Agron. AntillesGuyane, 1-2, 101-108.

Tessier D., Pedro G., 1980. Sur les propriétés de gonflement des argiles dans les sols. C.R. Acad. Sci., Paris, t. 291, Série D, 461-464.

Tessier D., Pedro G., Camara L., 1980. Sur le comportement hydrique et l'évolution de l'organisation des argiles (kaolinites et smectites) au cours de la dessiccation et de la réhumectation. C.R. Acad. Sci., Paris, t. 290, Série D, 1169-1172.

Thompson G. D., 1965. The relationship of potential evapotranspiration of sugar cane to environmental factors. Proc. ISSCT, (12), 3-9.

Valancogne C., Cabidoche Y.-M., Archer P., 1984. Premières conclusions des travaux de l'INRA sur l'irrigation de la canne à sucre (1980-1984). Ronéo, Station de Bioclimatologie et d'AgronomieScience du Sol, INRA-Antilles Guyane, 8 p. + annexes. 